\title{
DIE STAATSKLASSE/STAATSBOURGEOISIE IN DEN UNTERENTWICKELTEN LÄNDERN ZWISCHEN PRIVILEGIERUNG UND LEGITIMATIONSZWANG*
}

\author{
Von Hartmut Elsenhans
}

Zwei Haupttendenzen der gesellschaftlich-wirtschaftlichen Dynamik in der Peripherie lassen sich unterscheiden:

1. Die „Entwicklung von Unterentwicklung“ im Rahmen der Intensivierung der Integration der peripheren OKonomien in das Weltwirtschaftssystem, von der allerdings andere Autoren eine rapide Beschleunigung des Wirtschaftswachstums in den unterentwickelten Ländern erwarten mit der letztlichen Folge der Beseitigung von Massenarmut und Massenarbeitslosigkeit ${ }^{1}$.

2. Ein verstärktes Eingreifen staatlicher Organe in den ökonomischen Prozeß mit äußerst ambivalenter Zielsetzung (verstärkte Integration in oder Ausgliederung vom kapitalistischen Weltmarkt) ${ }^{2}$.

Im folgenden soll diese zweite Haupttendenz im Mittelpunkt von Überlegungen stehen, die weniger abgesicherte Ergebnisse referieren als zur Entwicklung von Hypothesen dienen sollen, die geeignet sein könnten, Forschungsanstöße zu geben.

\section{Zur Einordnung des Staatssektors in die Theorie des peripheren Kapitalismus und seiner Überwindung}

Trotz einer intensiven Diskussion über Staatsfunktionen im zentralen Kapitalismus fehlen $\mathrm{m}$. W. eingehende Untersuchungen über die Rolle des Staatsapparats in den unterentwickelten Ländern ${ }^{3}$. Vergleichende Untersuchungen über den Anteil des Staates am Bruttosozialprodukt, an der Kapitalbildung oder über das staatliche Interventionsinstrumentarium sind spärlich, sieht man von den für die Investitionstätigkeit multinationaler Unternehmen wichtigen Investitionshilfebestimmun-

\footnotetext{
* Uberarbeitete Fassung eines Referats für die Arbeitsgruppe Peripherie des Internationalen Systems der Deutschen Vereinigung für Politische Wissenschaft, Kongress Duisburg 7.-10. Oktober 1975.

$1 \mathrm{Im}$ Ưberblick: Senghaas, D. (Hrsg.): Imperialismus und strukturelle Gewalt. Frankfurt 1972; ders. (Hrsg.): Peripherer Kapitalismus. Frankfurt 1974; Frank, A. G.: Capitalism and Underdevelopment in (Hrsg.): Peripherer Kapitalismus. Frankfurt 1974; Frank, A. G.: Capitalism and Underdevelopment in
Latin America. Monthly Review Press. New York 1969, p. $150 \mathrm{ff}$; ; Cardoso, F. E.: Politique et développement dans les sociétés dépendantes. Anthropos. Paris 1971, v. a. p. 273 ff.; Dos Santos, Teotonio: Dependencia y Cambio Social. Cuadernos de estudios socio económicós. Universidad de Chile 1970, p. 15 ff.; Cordova, A.: Die wirtschaftliche Struktur Lateinamerikas. Frankfurt 1969; Sunkel, O.: El Subdesarollo latinoamericano y la teoría del desarollo. Siglo veintiuno editores. Mexico 1970 , p. $311 \mathrm{ff}$. Gegenposition der Notwendigkeit der Exportorientierten Industrialisierung: Balassa, B. et al.: The Structure of Protection in Developing Countries. John Hopkins. Baltimore 1971, p. 48 ff., p. $79 \mathrm{f}$.; Herman, ture of Protection in Developing Countries. John Hopkins. Baltimore 1971, p. 48 ff., p. $79 \mathrm{f} . ;$ Herman,
B.: The Optimal International Division of Labour. ILO. Genf 1975, p 2; UNCTAD. 3rd session. Santiago de Chile 13-4/21. 5. 1972. Bd. 1. Report and Annexes. UN. New York 1973, p. $181 \mathrm{ff}$.

2 Dos Santos, T.: Socialismo o fascismo. Prensa Latinoamericana. Santiago de Chile 1972, p. 32 f./90 ff.; Leff, Nathanael: Economic Policy Making and Development in Brasil. Wiley. New York 1968, p. 35 ff., p. 56.; Singh, B. D.: Economics of Development with Special Reference to India. Asia Publishing House, Bombay 1966, S. 536-540; Youngson, A. C.: Overhead Capital. A Study in Development Economics, Edinburgh Univ. Pr. Edinburgh 1967, p. 5-35; Hanson, A. H.: Le secteur public dans une économie en voie de développement. PUF. Paris 1961, p. 9; Belshaw, D. E. P.: Social and Economic Revolution for the Development of Backward Countries, in: Randall, L.: Economic Development Evolution or Revolution. Heath. Boston 1964, p. 8.

3 Green, P. R. H.: The Role of the State as an Agent of Economic and Social Development in the Least Developed Countries, in: J. of Development Planning 6/1974, p. 1-39; Hein, W./Simonis, G.: Entwicklungspolitik, Staatsfunktionen und Klassen. Auseinandersetzungen im peripheren Kapitalismus, in: Schmidt, A.: Strategien gegen Unterentwicklung. Frankfurt 1976, p. 216-249; Ianni, talismus, in: Schmidt, A.: Strategien gegen Unterentwicklung. Frankfurt 1976, p. 216-249; lanni,
O.: Estado e Capitalismo. Estructura Social e Industrialicaõ no Brasil. Editôra Civilizacaõ Brasileira. Rio de Janeiro. 1965 , v. a. p.18-22, p. 128, p. 185.
} 
gen und von den Investitionsplänen der Regierungen $a b$, aus denen sich die angestrebten Verbesserungen der allgemeinen Produktionsbedingungen ablesen lassen. Es gibt Anzeichen dafür, daß diese bedauerliche Lücke als Folge von drei Entwicklungen anzusehen ist:

- Konzentration der Imperialismustheorie auf den Nachweis der Deformation und Ausbeutung der Dritten Welt vor einer sozialistischen Revolution ${ }^{4}$,

- Konzentration der Dependenztheorie auf den Nachweis der Unmöglichkeit der Überwindung von Unterentwicklung gegen die "desarrollistas"5,

- Scheitern der modernisierungstheoretischen Ansätze als Folge ihrer Einbindung in die vom Zentrum vorgeschlagenen Entwicklungsstrategien, die einerseits nur in äußerst begrenztem Maße eine Abgrenzung gegenüber dem Weltmarkt akzeptieren und andererseits der Notwendigkeit gesellschaftlichen Strukturwandels deshalb nur Lippenbekenntnisse zollen, weil sonst die bisherigen Befürworter prowestlicher außenpolitischer Strategien ökonomisch geopfert werden müßten ${ }^{6}$.

Eine solche Nichtbeschäftigung mit dem Staat in der Dritten Welt steht im Widerspruch zu zwei Entwicklungen, die den peripheren Staat zu einem zentralen Untersuchungsgegenstand machen:

1. Auch im Rahmen der Entwicklung des peripheren Kapitalismus wächst der Staatssektor überproportional, und zwar sowohl im Bereich des unproduktiven Mehrproduktkonsums zur Absicherung von politisch als notwendig erachteten Brückenköpfen als auch in den "produktiven" Bereichen, d. h. in den Sektoren, die durch Herstellung von Gütern und Dienstleistungen (auch Infrastruktur) die Güterversorgung der Bevölkerung zu erhöhen suchen?. Dabei ist die Unterscheidung $\mathrm{zwischen}$ produktiv und unproduktiv problematisch:

Produktiv ist in einer kapitalistischen Wirtschaft die Herstellung von Gütern und Dienstleistungen die aufgrund der bestehenden Einkommensverteilung einen kostendeckenden Marktpreis erzielen. Wenn aber das Ziel des Entwicklungsprozesses die Beseitigung der gegenwärtigen Einkommensverteilung ist, dann kann längerfristig produktiv sein, was kurzfristig unproduktiv ist ${ }^{8}$. Aus der Überlegung, daß die vorhandenen Nachfragestrukturen nicht die Kriterien für entwicklungspolitische Entscheidungen liefern können ${ }^{9}$, die übrigens allen kolonialen Reformpolitiken der Zeit nach dem II. Weltkrieg zugrunde lag10, ergibt sich, daß (ohne Projektion einer alternativen Ökonomie) die "gewisse Libertät“ im Umgang mit den Marktgesetzen erlaubt,

- unter Berufung auf entwicklungspolitischen Zielsetzungen Ressourcen zu vergeuden,

4 Dos Santos, T.: 1970 , zit. in Anm. 1, p. $40 \mathrm{f}$

5 Dos Santos, T.: La crise de la théorie du développement et les relations de dépendance en Amérique latine, in: L'homme et la société, April-Juni 1969, p. 43-68, v. a. p. 61.

6 Gastiazora: Critica del Desarrollismo. Ed. Dos. Buenos Aires 1970, passim. Im übrigen wäre hier auf die Unmöglichkeit, effiziente Agrarreformen durchsetzen, zu verweisen.

7 Daten in: Bird, R. M./De Wulf, L. H.: Taxation and Income Distribution: A Critical Review of Empirical Studies, in: IMF Staff Papers 1973 (XX), p. 639-682; Quijano Obregon, Nacionalismo, neoimperialismo, y militarismo en el Perû. Ediciones Periferia. Buenos Aires 1971, p. 142;

8 Dies gilt z. B. für Investitionen in der Landwirtschaft, für deren Produkte es wegen der Unterbeschäftigung keinen Absatz gibt, genauso wie für Investitionen bei Investitionsgütern für die Landwirtschaft, vgl. dazu Falcon, W. P.: Agricultural and Industrial Interrelationsships in West Pakistan, in: Journal of Farm Economics, Dez. 1967, p. 1149;

9 Braun, O.: El desarrollo des capital monopolista en Argentina. Editorial Tiempo Contemporaneo. Buenos Aires 1970, p. $63 \mathrm{ff}$; Higgins, B. in: Echeverria, J. M./Higgins, B.: Social Aspects of Economic Development in Latin America. UNESCO. Paris 1963, p. 149; Vernon, R.: Manager in the Economic Development in Latin America. UNESCO. Paris 1963, p. 149; Vernon, R.: Manager in the
International Economy. Prentice Hall, Englewood Cliffs 1968, p. 164. International Economy. Prentice Hall, Englewood Cliffs 1968, p. 164.

10 Als hervorstechendes Beispiel die französische Reformpolitik in Algerien, Elsenhans: Frankreichs Algerienkrieg 1954-1962. München 1974 , p. $596 \mathrm{f}$. 
- unter Berufung auf entwicklungspolitischen Zielsetzungen Ressourcen zur Verbesserung des Produktionspotentials einzusetzen.

Wenn richtig ist, daß die Überwindung von Unterentwicklung die administrative Manipulierung des Marktmechanismus erfordert, weil die Binnenmärkte in den peripheren Gesellschaften im Unterschied zum Zentrum nicht einmal unvollkommen Massenbedürfnisse abbilden, dann wird der Ermessensspielraum der Staatsbürokratie sehr groß. Der Einwand, daß sie von den herrschenden Klassen kontrolliert wird, entkräftet diese These nicht, sondern spezifiziert sie weiter: Gerade wegen des Einflusses von einzelnen Mitgliedern der herrschenden Klassen besteht die Möglichkeit zur weiteren Vergeudung von Mehrprodukt (Lobbys, Familienclans etc.) $)^{11}$.

2. Parallel zur Verelendung breiter Massen und ihren politischen Folgen entstehen Konflikte innerhalb des Staatsapparats: Die enorme Privilegierung der handelskapitalistischen und grundbesitzenden Klassen in der Dritten Welt war und ist auch Folge der politischen Schwäche einer auf dem Lande in Subsistenzwirtschaft oder auch nicht lebenden Bevölkerung bisher noch geringen Organisationspotentials ${ }^{12}$. Mit der weltweiten Zunahme an Kommunikationsmöglichkeiten und der weiteren ökonomischen Verelendung auf dem Lande und in ihrer Folge in den Städten stellt sich die Frage, wie die gesellschaftlichen Hierarchien weiter abgesichert werden können. Dabei ist zu beobachten, daß die Staatsbürokratien - seien sie nun revolutionär (in der Folge von Befreiungskriegen, Algerien) durch Putsche (etwa in Peru) oder auch auf dem Weg des allmählichen Ausbaus des Staatssektors an die Macht gekommen - dazu tendieren, neben repressiven Maßnahmen Entwicklungsprogramme zu fördern, deren Ziel allgemein als Wirtschaftswachstum bezeichnet werden kann, das jedoch angesichts der unterschiedlichen Ausrichtung dieser Staatsklassen außerordentlich unterschiedliche Inhalte hat. Gerade über diese unterschiedlichen Inhalte der Entwicklungsplanung und über die gegenüber den Unterprivilegierten einzuschlagende Politik treten Konflikte innerhalb der Staatsbürokratien auf. Unter welchen Bedingungen Druck von unten zur Verstärkung der Repression von oben oder zur Akzentuierung der Entwicklungspolitik im Interesse breiter Massen führt, ist deshalb eine wichtige Frage für die politologische Forschung, zu deren Beantwortung noch viel zu geringe empirische Forschung geleistet wurde. Wenn das Interesse der herrschenden Klassen, des Auslandskapitals und der kapitalistischen Industrieländer an einer Stabilität der peripheren Systeme die Form der Repression und die Form der Entfaltung der Produktivkräfte im Interesse der Massen auch wenn nur in begrenztem Maße annehmen kann, dann erweist sich die These von der Funktion der Brückenköpfe in der Peripherie bzw. von der Funktion der Staatsbürokratien als Instrumente der Herrschaftssicherung als bloße Tautologie, mit der die spezifische Dynamik der peripheren Gesellschaften nicht erfaßt werden kann ${ }^{13}$.

11 Hussein M.: La lutte des classes en Egypte. Maspéro. Paris 1972, p. 201: „Dans ce secteur (des Staats), c'est le pot de vin qui constitue la forme principale d'accumulation “.

12 Lipton, Michael: Farm Price Stabilization in Underdeveloped Areas: Some Effects of Income Stability and Income Distribution, in: Streeten, P.: Unfashionable Economics. Weidenfeld \&Nicolson. London 1970, p. 23; Furtado, C.: What Kind of Revolution, in: Randall, 1964, Zit. in Anm. 2, p. 39. Flinn, W. L./Havens, A. E.: The Potential of Structural Change, in dieselben: Internal Colonialism and Structural Change in Columbia. Praeger, New York, 1970, p. 236; Raupp, P. M.: Land Reform and Agricultural Development, in: Southworth, H. M./Johnston, B. F.: Agricultural Development and Economic Growth. Cornell University Press. Ithaca 1967, p. 305.

13 Dos Santos, T., 1970, zit. in Anm. 1, p. 83; Qujano, A., zit. in Anm. 7, p. 90 und p. 184 ff. Caputo, O./Pizarro, R.: Desarrollismo y capital extranjero. Ediciones de la Universidad Tecnica del Estado. Santiago 1970, p. $10 \mathrm{ff}$.; Cardoso, F./Faletto, F.: Dependencia desarrollo en America Latina. 
2. Zwei Hinweise zum Zusammenhang zwischen der Frage nach der möglichen Rolle der Staatsklassen und den ökonomischen Bedingungen der Überwindung von Unterentwicklung

Es soll hier nicht die Ökonomie der Überwindung von Unterentwicklung eingehend analysiert werden, sondern nur auf zwei Zusammenhänge verwiesen werden ${ }^{\mathbf{1 4}}$, nämlich auf die Notwendigkeit langfristiger Planung zur Entwicklung von Produktivkräften und die Möglichkeiten der Aneignung von Renten ${ }^{15}$.

Die häufig geforderte Planung der wirtschaftlichen Entwicklung in den unterentwickelten Ländern beschränkt sich meist auf eine Kritik der Resultate von an Märkten orientierten Unternehmensentscheidungen in der Dritten Welt ${ }^{16}$. Die inhaltlichen Ziele einer Wirtschaftsplanung werden dabei nicht analysiert. Hier wird dagegen die These vertreten, daß nur über den Inhalt der Ziele der Planung, Ausmaß und Form (u. a. Zentralisierung - Dezentralisierung) erfaßt werden können. Dabei ist davon auszugehen, daß Armut und Unterbeschäftigung nur in dem Maße überwunden werden können, wie Massen für Massen produzieren ${ }^{17}$.

Eine Steigerung der Produktivität der Arbeit ist in den Branchen am leichtesten $\mathrm{zu}$ erzielen, die Massenprodukte herstellen, bei denen auch am leichtesten (wegen der Breite der Nachfrage) der Übergang zur Erzeugung der Produktionsmittel im unterentwickelten Land (einschließlich einer „angepaßten“ Technologie) möglich ist $^{18}$.

Der Übergang zu einer akkumulationsfördernden Einkommensverteilung mit relativ homogener Nachfrage setzt voraus, daß zuerst der Produktionsapparat schrittweise für die geplante Nachfragestruktur vorbereitet wird, weil sonst die Umverteilung nur zu Inflation oder steigenden Importen führt. Damit wird nicht behauptet, daß die Staatsbürokratien heute in der Dritten Welt dieses Ziel der Überwindung von Unterentwicklung über die Egalisierung der individuellen Nachfrage vorfolgen, sondern nur, daß allein eine Entwicklungspolitik, die auf Massennachfrage und Massenproduktion gegründet ist, Unterentwicklung überwinden kann und daß diese Politik eine relativ langfristige Planung zum Aufbau eines auf die Massennachfrage ausgerichteten Produktionsapparats erfordert ${ }^{19}$. Von hier aus ergeben sich Tendenzen zu seiner Stärkung der ökonomischen und der

Instituto de estudios peruanos. Lima. März 1967, p. 37; Worsley, P.: The Third World. Weidenfeld \& Nicolson. London 1964, p. 222; Ryelandt, B.: L'inflation en pays sous-developpé. Mouton. Paris/ Den Haag 1970, p. 64 ff.; Amin, Samir: Die ungleiche Entwicklung, Hamburg 1975, S. 273 ff. Dagegen Megahead, H. T.: Socialism and Nation Building in Africa. The Case of Mali 1968. Center for AfroAsian Research of the Hungarian Acadeny of Science. Budapest 1970, p. 9; Shivji, J. G.: Tanzania The Silent Class Struggle, in: Cliffe, L./Saul, J. S.: Socialism in Tanzania. East African Publishing House. Dar es Salaam 1973, p. $308 \mathrm{ff}$.

14 Dazu Elsenhans, H.: Die Uberwindung von Unterentwicklung, in: Nohlen, D./Nuscheler, F.: Handbuch der Dritten Welt. Hamburg 1975, p. 162-189.

15 Elsenhans, Hartmut (Hrsg.): Erdöl für Europa. Hamburg 1974, pp. 17 f. und 89-95.

16 Vgl. dazu Helleiner, G. K.: Socialism and Economic Development in Tanzania, in: Journal of Devel-

17 Vgl. Donges, Jürgen B.: Erfolgsbedingungen für binnenmarkt- und exportorientierte Industrialisierung in Entwicklungsländern, in: Giersch, Herbert/Haas, Heinz-Dieter: Probleme der weltwirtschaftlichen Arbeitsteilung. Berlin 1974, p. 37; zur Kritik der durchgeführten importsubstituierenden Industrialisierung; Sunkel, O.: Latin American Underdevelopment in the Year 2000, in: Bhagwati, J. N. L.: Economics and World Order, From the 1970's to the 1990's. Macimillan. London 1972, p. 205;

18 Exemplarisch Strassmann, W. P.: Economic Growth and Income Distribution, in: Quarterly Journal of Economics, Aug. 1956, p. 425-440; Sachs, I./Laski, K.: Industrial Development Strategy, in: Industrialisation and Productivity, Nr. 16, p. 44.

19 Vgl. z. B. die sehr kleinen Kapazitäten zur Düngemittelproduktion, die im Rahmen der importsubstituierenden Industrialisierung entstanden sind, obwohl wegen der Unterernährung in der Dritten Welt die Nahrungsmittelerzeugung die wichtigste Importsubstitutionsbranche wäre, Schuh, G. E.: Effects of Some General Economic Development Policies on Agricultural Development, in: Journal of Farm Economics, Dez. 1968, p. 1283. 
politischen Rolle der Staatsbürokratie, wenn zur „Systemstabilisierung“ immer effizientere Entwicklungsstrategien angegangen werden.

In diesem Prozeß ist zu erwarten, daß die Ressourcen der Staatsbürokratie v. a. im Handel mit den Industrieländern zunehmen. Ohne bestreiten zu wollen, daß Handel und Kapitalimporte durch die unterentwickelten Länder v. a. den Industrieländern nützen, die in den Genuß kostengünstiger Rohstoffe und mit niedrigen Löhnen produzierter Fertigwaren kommen, muß eingeräumt werden, daß in vielen Bereichen durch diese Wirtschaftsbeziehungen Differentialrenten entstehen, die unter den Bedingungen vollkommener Konkurrenz an die Verbraucher, unter den Bedingungen monopolitischer Kontrolle der Absatzmärkte durch multinationale Unternehmen diesen Konzernen zugute kommen ${ }^{20}$.

Im Erdölbereich hat die OPEC gezeigt ${ }^{21}$, wie solche Differentialrenten angeeignet werden können. Differentialrenten entstehen aber auch bei anderen Rohstoffen, und werden z. B. durch Exportsteuern (wie bei Kaffee oder Kupfer) durch die Produzentenländer angeeignet, wenn auch nur zu sehr kleinen Teilen ${ }^{22}$. Trotz der gegenwärtig dominanten Vorstellung, daß Erdöl ein spezifischer Rohstoff sei, ist davon auszugehen, daß im Fall der Monopolisierung durch die Angebotsländer auch bei anderen Rohstoffen die Aneignung von Differentialrenten (bei Kaffee auf der Grundlage des Konsumverhaltens des deutschen Arbeitnehmerhaushalts +700 Prozent, bei Tee +1450 Prozent) möglich sein dürfte ${ }^{23}$.

Da mit der Zunahme der innergesellschaftlichen Konflikte in den unterentwickelten Ländern die jeweils privilegierten Klassen v. a. die externen Einkünfte zu steigern versuchen werden (bei gleichzeitig verschärftem Nationalismus), dürfte als plausibel anzusehen sein, daß diese Möglichkeiten einer Kartellierung anderer Rohstoffe wahrgenommen werden ${ }^{24}$. Indem die Verlagerung von arbeitsintensiven und umweltverschmutzenden Branchen auf niedrigere Produktionskosten in der Peripherie zurückzuführen ist und in der Metropole die Umwelt- und Arbeitskosten solange weiter zu nehmen ${ }^{25}$, wie der zentrale Kapitalismus mit seinem entscheidenden Mechanismus - die Aneignung von Produktivitätsfortschritten durch die Masse der Bevölkerung in der Form von Reallohnsteigerungen bzw. in der Form der Bereitstellung verbesserter kollektiv konsumierter Güter (Umwelt) - weiter expandiert, sind auch im Bereich der ausgelagerten Produktionsschritte relativ rasch Differentialrenten zu erwarten.

Sowohl über die internationale Ebene der Organisation von Produzentenkartellen als auch bei der jeweils nationalen Aneignung von Differentialrenten wird inner-

20 Entweder generalisieren sich die günstigeren Produktionsbedingungen, und dann sinkt unter Konkurrenz der Preis auf Produktionskosten plus Durchschnittsprofit, oder ein wachsendes Angebot von Produzenten mit ungünstigeren Produktionsbedingungen wird nachgefragt, so daß die Produzenten mit günstigeren Produktionsbedingungen ein zusätzliches Einkommen über den Produktionskosten, die Differentialrente, erhalten. Tatsächlich war der Fall der Generalisierung der günstigeren Produktionsbedingungen selbst in der Phase des europäischen Hochimperialismus der normale Fall, vgl. Paish, G.: Great Britain's Capital Investment in Other Lands, J. of the Royal Society of Statistics, Sept. 1909, p. 475; ders.: Great Britain's Capital Investments in Individual Colonial and Foreign Countries, in: ebenda, Jan. 1911, p. 175.

21 Elsenhans, H./Werdé-Bräuniger, C./Wittgrebe, R.: Die Auseinandersetzungen zwischen den Förderländern und den Konzernen, in: Elsenhans, H.: Erdöl für Europa. Hamburg 1974, p. 91-95.

22 Braun, zit. in Anm. 9, p. 13 ff. UN Foreign Capital in Latin America, UN, New York 1955, p. 17/18, 26 und p. 65.

23 Elsenhans, H.: Okonomie der Rohstoffproduktion, in: Leviathan 1976/I, p. 122-129.

24 Zur Geschichte von Rohstoffabkommen im Ưberblick, Theess, W.: Kapitalistische Rohstoffmärkte Internationale Rohstoffabkommen, Berlin (DDR) 1967, p. 39-70; Radetzki, M.: International Commodity Agreements, Hurst, London 1970, UNCTAD, 3rd session, zit. in Anm. 1, Bd. IV, p. $24 \mathrm{ff}$.

25 Murray, R.: Underdevelopment, International Firms and the International Division of Labour, in: Towards a New World Economy. Universitaire Pers Rotterdam. Rotterdam 1972, p. 231; Elsenhans, H.I Junne, G.: Einige Aspekte des gegenwärtigen Wandels der internationalen Arbeitsteilung, in: Gantzel, J.: Herrschaft und Befreiung in der Weltgesellschaft, Frankfurt 1975, p. 240-266. 
halb der herrschenden Klassen der Dritten Welt die Staatsbürokratie gestärkt, da die Übertragung der Differentialrenten an die Unternehmen (soweit überhaupt durchführbar) bzw. bäuerlichen Kleinproduzenten sich für diese als Anreize zur Ausweitung des betreffenden Produktionszweiges abbilden würde, so daß die Produzenten dann die zu jeder Monopolisierung notwendige Produktionsbegrenzung unterlaufen würden ${ }^{26}$.

\section{Staatsbürokratie oder Staatsklasse als Klasse sui generis}

\subsection{Ambivalenz des Aufbaus einer Staatsklasse/Staatsbourgeoisie}

Der Aufbau einer Staatsbürokratie/Staatsklasse mit zunehmenden Interventionsmöglichkeiten im Produktionsprozeß ist keineswegs eine Garantie für die Überwindung von Unterentwicklung. Die Kritik am peripheren Kapitalismus und an den im Kolonialsystem entstandenen Wirtschaften hat gerade die Verprassung von Mehrprodukt durch die Aufblähung der staatlichen Verwaltungen hervorgehoben $^{27}$. Da die staatlichen Leistungen nicht an einem Markt bewertet werden, gibt es grundsätzlich keinen Nachweis für die Nützlichkeit von Leistungen außerhalb eines gesamtwirtschaftlichen Entwicklungsplans, der diese Leistungen der Produktionsentwicklung in den anderen Sektoren zuordnet, und nur geringe Möglichkeiten der Unterscheidung zwischen notwendigen Kosten zur Erbringung dieser Leistungen und unnötigen Ausgaben. Gerade in unterentwickelten Ländern mit Arbeitslosigkeit entsteht eine starke Tendenz im Staatssektor Personal aus Gründen nepotistischer Familienpolitik zu beschäftigen ${ }^{28}$.

Dies gilt auch für Staatsbetriebe. Zwar sind Staatsbetriebe anders als private Unternehmen auch dann an nationale Entwicklungsziele zu binden, wenn aus Investitionen keine Gewinne erzielt werden. Der Export von Kapital in der Form von Gewinntransfers aus Mangel an rentablen Anlagemöglichkeiten, wie ihn multinationale Konzerne praktizieren ${ }^{29}$, kann dadurch begrenzt werden. Zugleich können aber monopolistische Staatsbetriebe zu unrentabler Verschwendung tendieren, da ihr Management nicht durch die Konkurrenz auf Märkten kontrolliert und zu kostensparender Produktion gezwungen wird ${ }^{30}$. Genauso wird mit den im Rahmen langfristiger Entwicklungspläne durchgeführten Investionen erst Jahre nach Beginn der Vorhaben produziert. Inwieweit Kosten für die Steigerung der Produktivkräfte notwendig sind oder als Aneignung von Mehrprodukt durch die Kader anzusehen sind (und auch im Vergleich zum Rest der Gesellschaft privilegierte Belegschaften) läßt sich dabei nur durch betriebsinterne Überwachung feststellen,

\footnotetext{
26 Im Uberblick zu Marketing Boards: Abbott, J. C.: Agricultural Marketing Boards in Developing Countries, in: Journal of Farm Economics, Aug. 1967, p. $707 \mathrm{ff}$

27 Sabolo, Y./Gaude, J./Wery, R.: Les Tertiaires. ILO, Genf 1974, p. 36 und 57. Vgl. Touraine, A. u. A.: Emploi et sous-emploi en Amérique Latine, in: Cultures et Dévelopment 1968/1, p. 66.

28 Worsley 1964, zit. in Anm. 13, p. 109; Vgl. dazu Leff, N.: Economic Policy Making and Development in Brazil. John Wiley \& Sons. New York 1968, p. 135 ff.; Williams, G.: The Social Stratification of Neo-colonial Economy: Western Nigeria, in: Allen, Chr./Johnson, R. W.: African Perspectives. Cambridge Univ. Pr. Cambridge 1970, p. 241.

29 Vernon, R. 1968, zit. in Anm. 9, p. 209; Caputo, O./Pizarro, R., zit. in Anm. 13, p. 76;

30 Grimaud, in: Maghreb, Jan./Febr. 1972, p. 38 Bgl. auch die Stellungnahme von Präsident Boumediène, in: Actualités et Documents 5. 5. 1968, p. 6. El Djeich. Dez. 1972, p. 12-14, Rev.alg. des sc. jur. éc. et pol. Juni 1969, p. 489; Müller-Ohisen, L.: Möglichkeiten und Grenzen des Industriegüterexports der Türkei, in: Die Weltwirtschaft 1975 I, p. 130-132; Khatkhale, D. R.: Management in Entwicklungsländern, in: Finanzierung und Entwicklung, Sept. 1971, p. 10; Bhagwati, J. N./Desai, P.: Planning for Industrialization, Industrialization and Trade Policies since 1951. Oxford Univ. Pr. London 1970 , p. 157.
} 
zu der betriebsfremdes Personal schwer in der Lage ist. Ob Verschwendung unterbunden wird, hängt davon $a b$, inwieweit die Technokraten in den Betrieben und die Staatsbürokratie im Konflikt zwischen der Sicherung eigener Privilegien und der Notwendigkeit, sich gegenüber der Masse der Bevölkerung zu legitimieren, Selbstkontrolle üben. Die Anwendbarkeit des Instruments des Staatsbetriebes hängt von dem Niveau des Bewußtseins und dem Stand der gesellschaftlichen Auseinandersetzungen ab.

\subsection{Zum Problem einer neuen Form asiatischer Produktionsweise}

Der Widerspruch zwischen „Selbstbedienungsladen“ und effizientem Instrument der Förderung der Produktivkräfte über den Staatsapparat tritt historisch nicht nur in den Gesellschaften der Peripherie auf, sondern besteht in allen nichtkapitalistischen Klassengesellschaften mit Staatsapparat. Er war im Zentrum deshalb begrenzt, weil die Steigerung der Produktivkräfte schon in den frühkapitalistischen Phasen von einer Kapitalistenklasse getragen wurde, die im Kampf gegen vorkapitalistische Klassen die Aneignung von Mehrprodukt mit außerökonomischem Zwang zumindest im Kernland der industriellen Revolution relativ erfolgreich bekämpfte ${ }^{31}$. Wenn die Steigerung der Produktivkräfte heute in der Peripherie über den Staatsapparat erzielt werden muß, der einerseits eine akkumulationsfördernde Einkommens- und Nachfragestruktur herstellen müßte und dafür den Produktionsapparat im vorab ausrichten muß, so stellt sich die Frage nach dem Verhältnis zwischen dieser sich in einzelnen Ländern der Peripherie herausbildenden, in anderen Ländern erst anzeigenden Produktionsweise zu der sogenannten asiatischen Produktionsweise ${ }^{32}$.

Einige formale Parallelen über die Existenz einer Beamtenklasse mit ökonomischen Leitungsfunktionen hinaus sind sichtbar, so die Entkapitalisierung der Landwirtschaft durch Agrarreformen, deren Genossenschaften an die Dorfgemeinschaften der asiatischen Produktionsweise erinnern, nicht nur durch die kollektiv durchgeführte Produktionsplanung, sondern auch durch die Verknüpfung von Staat als Lieferant verbesserter Produktionsbedingungen (früher Be- und Entwässerung, heute Maschinen, Düngemittel und Bezahlung von Infrastrukturleistungen) und dörflicher gemeinsamer Planung oder die stärkere Präsenz der staatlichen Leitung im Bereich der gewerblichen Produktion (früher stark kontrollierte kleine Warenproduzenten oder Sklaven ${ }^{33}$, heute Staatsbetriebe mit „freier“ Lohnarbeiterschaft, wobei allerdings zu bedenken ist, daß auch früher selbst die unfreie Arbeitskraft gegenüber den Bauern materiell privilegiert war).

Von einer asiatischen Produktionsweise alten Typs unterscheiden sich diese Gesellschaften aber durch den Aufbau einer technologisch fortgeschrittenen Industrie, die zur Bildung einer Arbeiterklasse und einer technischen Intelligenz führt, und durch ihre Genese: Sie entsteht nicht durch die Möglichkeit der Anwendung außerökonomischen $\mathrm{Z}$ wangs seitens einer kleinen Klasse von Privilegierten gegenüber den

\footnotetext{
31 Dazu allg.: Roper, Trevor: The General Crises of the Seventeenth Century, in: Aston, Trevor: Crisis in Europe 1560-1660. Routledge \& Kegan, London 1965, p. $93 \mathrm{f}$.

32 Bester Überblick zur Diskussion der asiatischen Produktionsweise bei Chesneaux, J.: Le mode de

33 Wertheim, W. F.: Indonesian Society in Transition. A Study of Social Change. W. van Hove. Den Haag 1964, p. 2; Métraux, A.: Les Incas. Seuil. Paris 1961, p. 95 ff.
} 
Dorfgemeinschaften oder die Notwendigkeit der Durchführung relativ gleichbleibender Leitungsfunktionen seitens einer zentralisierten (oder von den Bauern gegen noch größere Ansprüche von privaten Grundherren zur Zentralisierung gezwungenen) ${ }^{34}$ Herrenklasse, sondern als Antwort auf die strukturellen Defekte eines peripheren Kapitalismus entweder in Abwehr gegen die über den Weltmarkt vermittelten Tendenzen zur Verschärfung von Unterentwicklung oder zur Stützung und Generalisierung der kapitalistischen Akkumulation im eigenen Land. In beiden Fällen ist die soziale Verelendung breiter Massen der Ansatzpunkt für ihre gesellschaftliche und politische Durchsetzbarkeit, weil ohne diese Verelendung offenbar keine Notwendigkeit bestünde, die Investitionsentscheidungen privater Unternehmer aufzuheben oder wenigstens durch kompensierende Maßnahmen zu ergänzen oder zu lenken.

Von den zwei Aspekten, die in der Diskussion über die asiatische Produktionsweise gegeneinander ausgespielt werden, nämlich Sicherung der Subsistenz bei Stagnation und despotischer Herrschaft bzw. relative Offenheit des Beamtenapparats nach unten und allmählicher Fortschritt der Produktivkräfte ${ }^{35}$, wäre bei den von den Staatsbürokratien dominierten Produktionsweisen der Peripherie heute der Aspekt des Wachstums und der relativ geringen Absperrung des politischen Systems im Vergleich zu den traditionellen asiatischen Produktionsweisen stärker repräsentiert. Zwar gilt auch im heutigen Erscheinungsbild die Beobachtung von Divitcioglu ${ }^{36}$, daß die Frage, inwieweit das verfügbare Mehrprodukt investiert oder verpraßt wird, nicht ökonomisch, sondern politisch determiniert ist, doch kann von der Hypothese ausgegangen werden, daß diese politische Bestimmung der Verwendung des Mehrprodukts erheblich mehr als früher vom internationalen Demonstrationseffekt und sehr viel schärferen innergesellschaftlichen Auseinandersetzungen bestimmt ist, mit der möglichen Folge einer beschleunigten Akkumulation. Die Kontroverse über den innovationshemmenden oder innovationserlaubenden Charakter der asiatischen Produktionsweise alten $\mathrm{Typs}^{37}$ müßte dann im Fall der neu sich herausbildenden peripheren Produktionsweisen in Richtung sogar auf den innovationsfördernden Charakter beantwortet werden.

Die Grundlage für dieses veränderte Verhältnis zum technischen Fortschritt ist in der Entwicklung der Eingliederung dieser Gesellschaften in die kapitalistische Weltwirtschaft zu suchen. Zunächst war in der Theorie der asiatischen Produktionsweise richtig analysiert worden, daß in dieser Produktionsweise anders als im europäischen Feudalismus durch die großen Möglichkeiten der Ausschaltung von Konkurrenz auf Märkten der technische Fortschritt nicht internalisiert, also nicht quasi-automatisch erzwungen wurde ${ }^{38}$. Dies gilt auch heute für die Gesellschaften mit weitgehender Verstaatlichung der industriellen Produktion in der Peripherie, sofern die Betrachtung auf die binnenwirtschaftlichen Beziehungen zwischen den Produktionseinheiten (Betriebe/Unternehmen/Kleinproduzenten) beschränkt wird.

34 Eberhard, W.: Geschichte Chinas. Von den Anfängen bis zur Gegenwart. Stuttgart 1971, p. 82 f.

35 Wittfogel, K. A.: Die orientalische Despotie. Eine vergleichende Untersuchung totaler Herrschaft. Köln/Berlin 1962, p. 183-188. Dagegen Ruben, W./Fischer, K.: Der Mauriya- und der Mogulstaat. Berlin (DDR) 1965, p. 22-24; Amin, 1975, zit. in Anm. 13, p. 42; Nguyen Khac Vien: Confucianisme et. Marxisme au Vietnam, in: Chesneaux, J./Boudarel, G./Hemery, D.: Tradition et Révolution au Vietnam. Anthropos. Paris 1971 , p. 23-34;

36 Divitćioglu, S.: Modèle économique de la société ottomane; Les XIV et XV siècles, in: La Pensée, April 1969, p. 56.

37 Die These der Rückständigkeit geht auf Marx zurück, MEW Bd. 9, p. 223. Dagegen: Chesneaux, J./ Bastide, M.: Des guerres de l'opium à la guerre franco-chinoise 1840-1885. Hattier Université. Paris 1969 , p. 24.

38 Amin 1975, zit. in Anm. 13, p. 52. 
Da aber die staatliche Übernahme von Leitungsfunktionen grundsätzlich mit dem Ziel des Aufholens gegenüber den Zentren verknüpft ist, spielt das Zentrum die Rolle des Innovationen erzwingenden Motors, weil nur über den kontrollierten wirtschaftlichen Fortschritt sowohl die vorhandene Deformation des Produktionsapparats korrigiert werden wie die über den internationalen Kommunikationsverbund ausgelösten steigenden Konsumerwartungen befriedigt werden können. Dabei ist einzuräumen, daß gerade über die Abhängigkeit der innovativen Wirtschaftslenkung der Staatsbürokratie vom politischen Ziel des Aufholens gegenüber dem Zentrum die Durchsetzung von Produktivkraftentfaltung bestimmt ist von den innergesellschaftlichen und internationalen Kooperations- und Konfliktmustern der Staatsklasse und nicht quasi-automatisch erfolgt.

Allerdings ist davon auszugehen, daß der in den frühkapitalistischen Phasen zu beobachtende Versuch der peripheren Staatsklassen, ihre Gesellschaften gegenüber den produktiveren Ökonomien des sich industrialisierenden Zentrums einfach abzuschließen ${ }^{39}$, heute aufgrund der Zerstörung der Subsistenzökonomie und der Deformation des Produktionsapparats nicht mehr möglich ist. Diese Abschließung war im 19. Jh. durch die „Öffnung der Märkte“ seitens der militärisch überlegenen kapitalistischen Mächte durchbrochen worden. Dabei entstanden über die Destabilisierung der peripheren Wirtschaften jene Konfliktpotentiale, die entstehenden neuen Eliten (den Führungskadern von Befreiungsbewegungen mit unterschiedlichen gesellschaftlichen Basen) bzw. traditionellen Eliten erlaubten, das Kräfteverhältnis zwischen dem Zentrum und der Peripherie in der Form der Abschaffung des Kolonialsystems und halbkolonialer Abhängigkeit auf der politischen Ebene entscheidend $\mathrm{zu}$ verändern ${ }^{40}$.

Während die Abgrenzung gegenüber den Ökonomien des Zentrums in der nachkolonialistischen Phase wieder leichter durchführbar geworden ist, besteht heute eine notwendige Verknüpfung zwischen Abgrenzung und Produktivkraftentwicklung, weil ohne das Ziel und in ihrer Effizienz differenziert zu beurteilende Maßnahmen der Produktivkraftentwicklung die Staatsbürokratie als politische Kraft sich gar nicht etablieren könnte. Um allerdings entscheiden zu können, in welcher Weise die Staatsbürokratien Prozesse der Erweiterung des Produktionspotentials in der Peripherie durchführen können, muß nach den spezifischen Bedingungen der Reproduktion einer Staatsklasse in der Peripherie gefragt werden.

\subsection{Die spezifischen Bedingungen der Reproduktion einer Staatsklasse/Staats- bourgeoisie in den peripheren Gesellschaften}

Besonders am Beispiel der Entwicklung des unabhängigen Algeriens, aber auch Schwarzafrikas wurde auf das Phänomen der Übernahme der privilegierten Stellungen der europäischen Kolonisation durch eine einheimische privilegierte Klasse verwiesen und dabei der Begriff der administrativen oder Staatsbourgeoisie ent-

39 Wilhelm, H.: Gesellschaft und Staat in China. Hamburg 1960, p. 67 ff.; Wills, J. E.: Pepper, Guns and Parleys. The Dutch East India Company and China 1622-1681. Harvard Univ. Pr. Cambridgel Mass. 1974 , S. $204-297$.

40 Zum widersprüchlichen Charakter der Befreiungsbewegungen Panikkar, K. M.: Asia and Western Dominance, Allen \& Unwin. London 1965 (4), p. $148 \mathrm{ff}$., p. $238 \mathrm{ff}$; Halstead, J. P.: Rebirth of Nation. The Origins and Rise of Moroccan Nationalism 1912-1944. Center for Middle Eastern Studies. Harvard Univers. Pr. Cambridge (Mass.) 1967, p. $165 \mathrm{ff} . ;$ Kahin, George Mac Turnan: Nationalism and Revolution in Indonesia. Cornell Univers. Pr. Ithaca 1952, p. 49. 
wickelt ${ }^{41}$. Diese Begriffe (Chaliand, Fanon, Leca, Arrighi) haben einen für diese Diskussion wichtigen politischen Inhalt, nämlich die Denunzierung sich als sozialistisch ausgebender Systeme als durch Ungleichheit und ökonomische Privilegien bestimmte.

Die Bezeichnung einer sich herausbildenden privilegierten Klasse als Bourgeoisie verstellt aber die spezifischen Reproduktionsbedingungen einer Bourgeoisie und einer Staatsklasse. Eine Bourgeoisie ist dadurch gekennzeichnet, daß ihre Mitglieder als Besitzer von Produktionsmitteln auf Märkten konkurrieren und nur solange Mitglieder der herrschenden Klasse bleiben, als sie aufgrund der innovativen Anlage des auf sie entfallenden Anteils am Mehrprodukt konkurrenzfähig bleiben. Die Bourgeois sind auf Gedeih und Verderb an die Rentabilität ihrer Investitionen gebunden und können sich, was hier nicht abgeleitet werden kann, ökonomisch nur behaupten, insoweit über den Widerstand der abhängigen Arbeitskraft wachsende Binnenmärkte die Erweiterung der Produktion durch Arbeitsteilung und modernere Technologie "rentabel“ werden lassen ${ }^{42}$. Da genau dieser gesellschaftliche Mechanismus, der im zentralen Kapitalismus den Ausgleich zwischen der Produktions- und Konsumtionskapazität herstellt, in der Peripherie fehlt ${ }^{43}$, hat sich nur eine vom Zentrum auch nach Abgrenzungsversuchen wieder abhängige „Lumpenbourgeoisie“ 44 bilden können, mit starken Refeudalisierungstendenzen (Nitsch) ${ }^{45}$.

Für eine Staatsklasse gilt jedoch gerade die Forderung nach (kurzfristiger) Rentabilität aufgrund einer gegebenen Nachfragestruktur nicht. Mitglied der Staatsklasse bleibt man nicht, weil die eigenen Entscheidungen zur Rentabilität der Investitionen führen, sondern weil einerseits die gesamte Klasse legitim bleibt, d. h. ein (nach jeweils erreichtem Stand der Klassenauseinandersetzungen jeweils unterschiedliches) $\mathrm{Maß}$ an Massenloyalität sichern kann und weil jedes einzelne Mitglied sich über die sich herausbildenden Clans und Cliquen bei der Jagd auf Posten und Pfründen einen Platz sichern kann. Dabei ist die Annahme, daß Mitglieder der Staatsbürokratie, die einen großen Beitrag für die Entwicklung der Produktionskräfte geleistet haben, mit im System internalisierten „Belohnungen“ rechnen können, keineswegs notwendig46.

Die Frage zur Ausrichtung der Wirtschaftspolitik der Staatsbürokratie muß vielmehr ausgehend von der politökonomischen Basisrationale, die überhaupt zu ihrer Bildung geführt hat, beantwortet werden. Da die Rekonstituierung von Staatsklassen in der Peripherie als Folge der für die Massen unzureichenden kapi-

\footnotetext{
41 Leca, J.: Capitalisme d'Etat, Socialisme, Nationalisme en Algérie, in: Rev. alg. des sc. jur. éc. et pol., März 1968, p. $251 \mathrm{ff}$.; Kaid, Ahmed: Contradictions de classes et contradictions au sein des masses, in: Rev. alg. des sc. jur. éc. et pol., Dez. 1970, p. 1083; Chaliand, G.: L'Algérie est-elle socialiste. Maspéro. Paris 1964, p. 20 ff.; Fanon, F.: Sociologie d'une révolution. Maspéro. Paris 1968, p. 96-103; Arrighi, G./Saul, J. S.: Socialismo e sviluppo economico nell'Africa tropicale, in: Arrighi, G.: Sviluppo Economico e Sovrastrutture in Africa. Einaudi. Turin 1969, p. 251-265. Vgl. auch Thoden van Elzen, H. U. E.: Staff, Kulaks and Peasants: A Study of a Political Field, in: Cliffe, L./Saul, J. 1973, zit. in Anm. 13, p. 158. Boumediène, zit. in: Jeune Afrique 22. 6. 1976, p. 27 und in: Révolution Africaine 2./8. 3. 1973, p. 8; Caputo, O./Pizarro, R., zit. in Anm. 13, p. 10 f.; Riad, H.: L'Egypte Nassérienne. Minuit. Paris 1964, p. 90. Hussein, M., 1971, zit. in Anm. 11, p. $195-204$.

42 Tavares, M. C.: Substitution d'importations et développement économique en Amérique latine, in: Martins, L.: Amérique latine, Crise et dépendance. Anthropos, Paris 1972, p. 52-64.

43 Singer, H. W.: US Foreign Investment in Underdeveloped Areas. The Distribution of Gains between Investing and Borrowing Countries, in: American Economic Review, Mai 1950, p. $478 \mathrm{ff}$.

44 Frank, André Gunder: Lumpenbourgeoisie et Lumpendéveloppement. Maspéro. Paris 1972.

45 Nitsch, Manfred: Legitimationsprobleme in der Dritten Welt - Ausgehend vom Brasilianischen Modell. DVPW Okt. 1975 , p. 27.

46 Dos Santos 1972, zit. in Anm. 2, p. $36 \mathrm{ff}$; Myrdal, G.: The 'Soft' State in Underdeveloped Countries, in: Streeten, P., 1970, zit. in Anm. 12, p. 234-239; Scott, J. C.: The Analysis of Corruption in Developing. Countries, in: Comparative Studies in Science and History 1969, v. a. p. $324 \mathrm{f}$. und die in Anm. 41 zit. Literatur.
} 
talistischen Entwicklung in der Peripherie interpretiert wurde, ist davon auszugehen, daß die Reproduktion der Staatsklasse davon abhängt, inwieweit sie über Repression und Konzessionen das bestehende System stabilisieren kann. Die (z. T. von den Theoretikern der antisubversiven Kriegsführung in der französischen Algerienarmee ausgebildeten) peruanischen Militärs sind hier ein illustratives Beispiel ${ }^{47}$ : Als die campesions zum bewaffneten Widerstand übergingen, hat die peruanische Oligarchie ihre Armee zur Repression in die Aufstandsgebiete geschickt, die dort feststellen mußte, daß nur ökonomische und soziale Reformen das Problem lösen konnten. Die Folge war ein Putsch, durch den sich das Militär zur Staatsklasse konstituierte, ökonomische Leitungsfunktionen übernahm und in höhere Einkommenspositionen vorrückte.

In dem Maße, in dem die Staatsklasse Legitimität produzieren muß, wird sich folgender Prozeß abspielen: Die verschiedenen in Clans und Cliquen aufgespaltenen Segmente der Staatsbürokratie konkurrieren um Einfluß, Privilegien und Machtchancen innerhalb des Staatsapparats. Die Durchsetzungsmöglichkeiten hängen einerseits von der internen Struktur der bürokratischen Institutionen und der Staatsklasse $a b$, doch gibt es unter der Voraussetzung der Notwendigkeit, Stabilität des Systems zu produzieren, im Fall unzureichender Resultate für die verschiedenen Klassen stets die Möglichkeit für einzelne Mitglieder und Cliquen, sich in Allianz mit „unzufriedenen“ Klassen größere Machtchancen und Privilegien zu sichern. Dies erklärt die beobachtbaren Tendenzkämpfe in den Staatsbürokratien. Eine typische Konstellation bei sich verstärkendem Druck von unten ist der Versuch der subalternen Grade (die häufig zitierten Hauptleute) ${ }^{48}$, die im Kontakt mit der sozialen Realität die unzureichenden Ergebnisse der staatlichen Politik als Behinderung der Durchführung des ihnen übertragenen Auftrags, lokal Stabilität zu produzieren, erleben, durch einen „Machtwechsel“ die Politik zu verändern und ihre Einkommen zu erhöhen. Die andere Konstellation ist die Beantwortung des Drucks von unten durch verschärfte Repression in Allianz mit den privilegierten Klassen bzw. der Verzicht auf Reformen, wegen fehlenden Drucks von unten, durch dessen Ausbleiben die Staatsbürokratie fast ausschließlich von den meist dauerhafter organisierten und über kürzere Einflußwege verfügenden privilegierten Klassen bearbeitet wird ${ }^{49}$.

Deutlich wird hier, daß die Staatsklasse durch spezifische Konkurrenzbeziehungen gekennzeichnet ist, durch die sich die gesellschaftlichen Auseinandersetzungen in der Form der Verteilung von Machtchancen und Privilegien der einzelnen Segmente der Staatsklasse durchsetzen. Dabei kann kein direkter Bezug zwischen der Ausrichtung der Staatsklasse und dem Stand der Klassenauseinandersetzung hergestellt werden. Entsprechend der unterschiedlichen Formen der Systemstabilisierung, Reform oder Repression, kann verstärkter Druck von unten zu verstärkter Repression oder Orientierung der Entwicklung der Produktivkräfte am Interesse der Massen führen, der Verstärkung von Strategien des sogenannten „nichtkapitalistischen" Entwicklungsweges ${ }^{50}$.

\footnotetext{
47 Alonso, Enricque: Fuerzas armadas y revolución nacional en Bolivia y Perú, in: Estrategia, Jan./Febr.

48 Bopegamage, E.: The Military as a Modernizing Agent, in: Economic Development and Cultural Change, Okt. 1971, p. 71-79. Klassen und Klassenkampf in den Entwicklungsländern, Berlin (DDR) Bd. 1, 1969, p. 268. Chesneaux, J.: Geschichte Ost- und Südostasiens im 19. und 20. Jh., Köln 1969, p. 195. Am Bsp. Dahomeys, Renke, Ludger: Die Eingriffe des dahomeischen Militärs in die Politik, in: Probleme der Entwicklungsländer 48/1972, p. 147.

49 Grecic, V.: Trasfondos del Golpe de Estado en el Ecuador, in: Estrategia, März/April 1972, p. 53-56.

50 Horowitz, I. L.: Military Elites, in: Lipset, S. M./Solari, A.: Elites in Latin America. Oxford Univ. Pr. New York 1967, p. 183.
} 
Die spezifische Reproduktionsdynamik der Staatsbürokratie rechtfertigt, sie als Klasse sui generis zu bezeichnen (auch wenn sie auf Bündnisse mit anderen Klassen angewiesen ist), die Ungeklärtheit der Formen der Systemstabilisierung hat aber zur Folge, daß von einer eindeutigen Ausrichtung ihrer Wirtschafts- und Gesellschaftspolitik nicht auszugehen ist. Vielmehr hat gerade ihre Anbindung an eine solche allgemeine Zielsetzung zur Folge, daß die von den politischen Strukturen und den vorherrschenden bzw. vorhandenen soziokulturellen Normensystemen der Staatsbürokratie und der übrigen Klassen beeinflußten Durchsetzungsmöglichkeiten für repressive und reformerische Strategien auf die interne Konkurrenz innerhalb der Staatsklasse und ihre politische Ausrichtung zurückwirken.

\section{Bedingungen möglicher Szenarios}

Für die Ausrichtung der Staatsklassen müssen also neben dem Stand der Klassenauseinandersetzungen - ohne die Befürchtung vor systemgefährdendem Widerstand der Unterprivilegierten ist grundsätzlich nicht mit einer Politik der Überwindung von Unterentwicklung im Interesse der Unterprivilegierten zu rechnen - die interne Struktur der Staatsklasse und die vorherrschenden soziokulturellen Normen, die Form des politischen Willensbildungsprozesses, die institutionelle Verfaßtheit des Entscheidungs- und Meinungsbildungsprozesses mit ihren Auswirkungen auf die Möglichkeiten zur Bewußtmachung, Artikulation und Vertretung von Interessen der verschiedenen Klassen untersucht werden. Es bestehen Hypothesen über mögliche Entwicklungen, die sich an Hand von zwei Alternativpaaren, nämlich der Höhe des von außen zu erwartenden Einkommens und der Ausrichtung des Staatsapparats in folgende Idealtypen aufgliedern lassen.

\begin{tabular}{lcc}
\hline & \multicolumn{2}{c}{ Staatsapparat handelt überwiegend } \\
Höhe des äußeren & im Interesse & im Interesse \\
Einkommens & der Privilegierten & der Massen \\
- hoch & I Vorwiegend & II technokratische \\
& sektorales Wachstum & Entwicklung \\
& mit Entwicklungs- & \\
& stopp & \\
- niedrig & III Verschärfung von & IV langsame bäuerliche \\
& Unterentwicklung & Entwicklung \\
& mit geringem & \\
& Wachstum & \\
\hline
\end{tabular}

Ein hohes äußeres Einkommen über die Aneignung von Differentialrenten wird nur noch in wenigen Fällen zu bloßen Umverteilungsmaßnahmen (begrenzten oder weniger begrenzten Ausmaßes) führen, wie etwa in Teilen der Ölscheichtümer am Golf. Statt dessen wird Technologieimport und die Subventionierung der Industrialisierung betrieben, deren Effekte auf dem Arbeitsmarkt aller Voraussicht nach zu klein bleiben dürften, als daß die Masse der Bevölkerung als Arbeitskraft 
in den modernen Sektor integriert würde und sich über Lohnkämpfe einen steigenden Lebensstandard sichern könnte ${ }^{51}$.

Stammen die äußeren Einkommen aus Kapitaltransfers (etwa durch den Kapitalimport für die verarbeitende Export- oder auch in einzelnen Fällen binnenmarktorientierte Industrialisierung), so führt die fehlende Ausweitung der Märkte bzw. die ausbleibende Konkurrenzfähigkeit bei im Vergleich zu anderen Niedriglohnländern zu schnell steigenden Löhnen zum Retransfer von Gewinnen, einem Nettoabfluß von Kapital ${ }^{52}$.

Sind die äußeren Einkommen niedrig, so wird der für die oben skizzierten Umverteilungsmaßnahmen verwendbare Surplus klein sein und Wachstum weiter beschränkt, ohne daß die Privilegierten im Rahmen der Eingliederung in das Konsummodell des „Kosmopolitanen“ Weltsystems (Nitsch) ${ }^{53}$ darauf verzichten, durch Lohnsenkungen ihren Anteil am Gesamtprodukt für die Finanzierung ihres mit der Einkommensentwicklung im Zentrum steigenden Konsums zu erhöhen.

Im Fall eines niedrigen äußeren Einkommens und einer Entmachtung der privilegierten Klassen muß allein durch die Landwirtschaft das Mehrprodukt für jene Arbeitskräfte erwirtschaftet werden, die für die Vorbereitung der industriellen Produktion eingesetzt werden. Ohne große Devisenreserven bleibt der Technologieimport klein ${ }^{54}$. Es entstehen nur wenige hochqualifizierte Industriearbeiter und Kader, die hohe Konsumnormen durchsetzen könnten, so daß sie politisch relativ kontrollierbar sind. Das Entwicklungstempo bleibt relativ langsam und die sozialen Spannungen eher begrenzt.

Wenn die äußeren Einkommen hoch sind - und dies wurde oben als für sehr viele Rohstoffe exportierende Länder angenommen und gilt (weil auch für Länder mit arbeitsintensiven verarbeitenden Branchen mittelfristig wegen des Auftretens von Differentialrenten anzunehmen) für alle in engerem Wirtschaftsaustausch mit den Industrieländern stehenden unterentwickelten Länder -, dann kann eine an den Interessen der Massen sich ausrichtende Staatsbürokratie eine rasche Modernisierung des Produktionsapparats in einer sektorial abgestimmten Entwicklung der Produktivkräfte erreichen. Voraussetzungen sind eine Agrarrevolution nach dem Aufbau der Betriebe, die die für die Steigerung der Hektarerträge notwendigen Inputs herstellen, die Orientierung an dem bei steigenden Realeinkommen zu erwartenden Massenkonsum und die Reservierung der Devisen für jene Produkte, mit denen die eigene Produktionsmittelproduktion gesteigert werden kann, um unterbeschäftigte Arbeitskräfte mit Produktionsmitteln auszurüsten bzw. aus bisher wenig produktiven Sektoren durch Steigerung der Arbeitsproduktivität Arbeitskräfte abziehen zu können, die für wachsende Massenbedürfnisse produzieren ${ }^{55}$. Dieser letzte Fall ist für die Theorie am interessantesten: Einerseits entsteht über den Technologieimport eine technische Intelligenz und eine qualifi-

51 Zur Arbeitsplatzentwicklung: Hervé, M.: Employment and Industrialization in Developing Countries, in: Quarterly Journal of Economics, Febr. 1966, S. $104 \mathrm{ff}$.

52 Die ergibt sich aus den insgesamt geringen Märkten auch für verarbeitete Produkte in den Industrieländern, Dorner, K.: Probleme einer weltwirtschaftlichen Integration der Entwicklungsländer, Tübinländern, Dorner, K.: Probleme einer weltwirtschaftlichen Integration der Entwicklungsländer, Tübin-
gen-Basel 1974 (2), p. 159. Eine Form sind z. B. Lizenzgebühren, die rasch wachsen (Mexiko 1953-1968 gen-Basel 1974 (2), p. 159. Eine Form sind z. B. Lizenzgebühren, die rasch wachsen (Mexiko 1953-1968
$19 \%$ pro Jahr, Argentinien 1965-1970 $27 \%$ pro Jahr, Südkorea $44 \%$ pro Jahr) und im Fall Mexiko 1968 15,9\% (Argentinien $19697,9 \%$ ) der Exporterlöse kosteten. UNCTAD, 3rd session, zit. in Anm. 1, Bd. III, p. 115-116.

53 Nitsch, M.: Das brasilianische Modell: Ende eines Wirtschaftswunders, in: Aus Politik und Zeitgeschichte $35-36 / 7-6,28.8$. 1976 , p. 26.

54 Zum Beispiel Tanzanias: Ngotyana, B.: The Strategy of Rural Development, in: Cliffe/Saul 1973, zit. in Anm. 13, p. 122.

55 Elsenhans, H.: Die ökonomische Funktion der Argrarrevolution zur Überwindung von Unterentwicklung, in: Internationale Entwicklung 1975/I, p. 35-37. 
zierte Arbeiterschaft, die die sektoral steigende Produktivität zu Privilegierungsansprüchen auszunutzen suchen und damit die Privilegierungstendenzen der Staatsbürokratie stützen ${ }^{56}$.

Zugleich bedeutet ein Abhängen des Rests der Bevölkerung, daß kein ausreichender Binnenmarkt entsteht, mit der Folge einer neuen ungleichen Eingliederung in das kapitalistische Weltsystem. Inwieweit die mit großen Planungskompetenzen aber häufig geringen internen Kontrollinstanzen (geringe administrative Kompetenz) ausgestatteten modernistischen Staatsbürokratien den Vorrang nicht auf die eigene Priviligierung (und die der technischen Intelligenz und der Arbeiterschaft) legen, hängt neben dem Druck von unten - d. h. u. a. der Vermittlung zwischen zentraler Planung und Partizipation auf gemeindlicher Ebene - davon ab, inwieweit

- die Strukturveränderungen auf dem Lande gelingen

- die Privilegierung der Arbeiterschaft und der technischen Intelligenz durch nicht-ökonomische Leistungsanreize in Grenzen gehalten werden können

- die Staatsklasse aufgrund ihrer Rekrutierung, einer geringen Versäulung (scharfe Konkurrenz um Legitimität, Offenheit nach unten) und ihrer wirtschaftspolitischen Erklärungsmuster einen Prozeß der Produktivkraftentwicklung durch Eingliederung der Masse der Bevölkerung in einen im Interesse der Masse der Bevölkerung organisierten Produktionsprozeß durchhalten kann.

$\mathrm{Da}$ in einem durch politische Auseinandersetzungen bestimmten Entwicklungsprozeß die Erwartungen über die Ergebnisse entwicklungspolitischer Strategien selbst eine große Rolle spielen, hat hier die entwicklungspolitische Diskussion in der Metropole eine nicht unerhebliche Bedeutung. Solange nämlich hier die Überwindung von Unterentwicklung von einer veränderten Exportspezialisierung, diesmal auf arbeitsintensiv hergestellte Produkte der verarbeitenden Industrie, erwartet wird, die nur zur Folge hat, daß angesichts der Arbeitsmarktstruktur in der Peripherie neben Bananen und Kaffee eben auch Oberhemden etc. zu Niedriglohnprodukten werden, wird der privilegierte Teil der Bevölkerung mit dem Hinweis auf dieses Argument den notwendigen gesellschaftlichen Strukturwandel ablehnen. Und wer vermöchte sich nicht vorzustellen, daß die in der Metropole vorgebrachte Argumentation, daß im Entwicklungsprozeß sich die Einkommensungleichheit zwangsläufig verschärft, auf bewundernswerte Weise denen zustatten kommt, die hohe Profite, Beamtengehälter und hohe private Renteneinkommen verteidigen, die sie allerdings keineswegs investieren, sondern unproduktiv konsumieren bzw. v. a. soweit es sich um kapitalistische Unternehmen handelt, exportieren.

56 Elsenhans, H.: Der algerische Weg der Ưberwindung von Unterentwicklung, in: Leggewie, C./Nikolinakos, M.: Europäische Peripherie. Dritte Welt, Sonderheft 1975, Meisenheim 1975, p. 225-232; Shahid, Javed Burki: A Study of Chinese Communes, in: Chen, K. I./Uppal, J. S.: Comperative Development of India and China. Free Press/Collier Mac Millan. London 1971, p. $179 \mathrm{f}$;; Chen, N. R./Galenson, W.: The Chinese Economy under Communism. Aldine Publishing Corp. Chicago 1968, 153 ff.; Howe, Chr.: Wage patterns and Wage Policy in Modern China 1919-1942. Cambridge University Press. Cambridge 1973 , p. $137-141$. 


\title{
The Ideological Orientation of the New States
}

\author{
BY Herbert Krüger
}

The ideological orientation of the new states can derive from their own values and traditions or from an intellectual exchange with "East" and "West". After the end of colonialism, the former attitude could be expected, but the new states' desire to "modernize" turns their view to the examples of East and West. It is, however, doubtful whether these old continents have much to offer, as they are themselves in need of renewal and their rigid, quasireligeous ideologies are not too inviting for countries looking for new ways. Despite these shortcomings of East and West, there are only few countries attempting a complete return to their own roots. Most of the time, authenticity is restricted to superficialities like names. More important are attempts at a "Third Way". The various organizations establishing co-operation among the developing countries which have made their impact in International Law and ideologies like "Arab", "African" etc. . . "Socialism" are important in this respect. With regard to intellectual communication between the West and the Third World, there are not many Western theoreticians with major impetus (Marx being introduced as an representative of "Eastern" thought). Comte was influential in Latin America, Bentham in India, but on the whole, the West has been more successful in exporting institutions rather than ideologies. The most important such institution is the "state", though in many countries of the Third World it has become the instrument of a small elite instead of an institution of general welfare.

The same is true for "democracy", to which almost all countries pay lip-service without adopting such essential elements as free elections between alternatives, freedom of opposition (elements which also in the West needed centuries to develop). A western heritage of doubtful value, finally, is the pursuit of welfare and affluence, monopolized by a small elite but aspired to by all. It remains to be studied, how systems subscribing to socialism and austerity handle these problems, and how much they are influenced by the "East" or going their own way.

\section{The State Class/Administrative Bourgeoisie in Underdeveloped}

\section{By Hartmut Elsenhans}

The developing countries will be able to appropriate the differantial rents and the consumer rents from their raw material exports (and later on from their exports of simple processed articles). Overcoming underdevelopment will no longer be hampered by lack of foreign currency or investible funds, but nevertheless will not be possible automatically by the disposal of funds. Under this assumption the internal societal dynamics of underdeveloped countries become a problem of primordial concern.

The preceding article tries to give new hints for dealing with the tendencies of increasing state participation in economic development of the LDC's. Considering that the sharpening social crisis in the LDC's forces both governments which 
try to keep peripheral capitalism going, and governments that come to power by popular movements promoting alternative ways of economic development, to "do something for the underprivileged masses", the article rejects the simplistic notion of the state as a modernizing agent. On the contrary, the concept of bureaucracy as a state class comparable to the state classes of the so-called "asiatic modes of production" should be further elaborated. The central approach is, that a state class does dispose of the mass of economic surplus of society and in opposition to the false notion of administrative bourgeoisie - is not forced by mechanisms of economic competition to invest this surplus in oder to increase productive forces.

The tendencies of formation of a state class with these caracteristics are a necessity in LDC's, because existing markets do not indicate rational choices for investment as income distribution is highly unequal. But the potential benefit of the existence of a state class, that is the possibility of investing funds in order to promote productive forces in the interest of a later increase of mass consumption, is not necessarily realised. The state class can also use the economic surplus for its own conspicuous consumption.

Whether the state class wastes funds or whether it invests funds for mass needs, is not economically determined, but is the outcome of social conflicts influencing the specific mechanisms of the internal rivalries of the state class. The article gives some hints, in which direction these societal dynamics should be further explored.

The article is a further investigation into the political and social dynamics of overcoming underdevelopment the basic economic stratagem of which has been presented in my article on: "Overcoming Underdevelopment. A Research Paradigm," published in the Journal of Peace Research No. 4 Vol. XII/1975, pp. $293-313$.

State and class in peripher-capitalistic societies: the development of dependent state-capitalism in Black Africa

\section{By Rainer TetzlafF}

The article deals with two main scientific issues: how can the function of the state in the development process of LDC be defined, and which are the main classes in peripher-capitalistic societies. Some authors qualify the state in LDC as politically weak, others as relatively strong and independent from social classes. The article stresses the fact that today some important state functions are fulfilled by international organisations; that is why one can formulate the thesis of an internationalisation of state functions in LDC.

In the second part follows a discription of the historical genesis of state functions and class formations in Africa during the period between 1880 and 1960. Five types of colonies can be distinguished, each of them being characterized by specific colonial interests and specific "classes" (migrant workers, cash crop-peasants, mine workers etc.) as the result of colonial rule.

The colonial state had as a main function the dissolution of pre-capitalistic conditions for production. At the eve of independence a bureaucratic and urbanized local petty bourgoisie inherited the colonial state apparatus which 